\title{
Fuzzy Topological Transformation Groups
}

\author{
Tazid Ali(Corresponding author) \\ Department of Mathematics, Dibrugarh University \\ Dibrugarh 786004, Assam, India \\ E-mail: tazidali@yahoo.com \\ Sampa Das \\ Department of Mathematics, Dibrugarh Polytechnic \\ Dibrugarh, Assam, India
}

\begin{abstract}
In this paper we have introduced the concept of Topological Transformation Groups in fuzzy setting as a natural transition from the corresponding crisp structure and study some properties thereof. Classical results on orbits, orbit closure, invariant subsets are investigated in this setting. Finally we have constructed some new fuzzy topological transformation groups from given ones.
\end{abstract}

Keywords: Fuzzy topological transformation group, Fuzzy orbit

\section{Introduction}

A classical Topological Transformation Group is a structure $(\pi, \mathrm{G}, \mathrm{X}$,$) where \mathrm{G}$ is a topological group, $\mathrm{X}$ is a topological space and $\pi$ is a continuous function from $G \times X \rightarrow X$ satisfying $\pi(0, x)=x$ and $\pi(s, \pi(t, x))=$ $\pi(s+t, x)$, where 0 is the identity of $\mathrm{G}$. In this paper we fuzzify the above concept as a natural transition from the corresponding crisp structure. For this fuzzification we will consider a fuzzy topological group (Chu-hai Yu, 1987), a fuzzy topological space and a fuzzy continuous map from $G \times X \rightarrow X$ satisfying the above stated conditions. Throughout our discussion the fuzzy topology on any set will contain all the constant fuzzy subsets. In other words we will use Lowen (R. Lowen, 1976) definition of fuzzy topology.

\section{Preliminaries}

In this section we recall some preliminary definitions and results to be used in the sequel.

Let $\mathrm{X}$ be a non-empty set. A fuzzy set in $\mathrm{X}$ is an element of the set $I^{X}$ of all functions from $\mathrm{X}$ into the unit interval I. A fuzzy point of a set $\mathrm{X}$ is a fuzzy subset which takes non-zero value at a single point and zero at every other point. The fuzzy point which takes value $\alpha \neq 0$ at $x \in X$, and zero elsewhere is denoted by $x_{\alpha}$. If $x \in X$, then the fuzzy point $x_{1}$ will be denoted simply by x. Let $\lambda$ be a fuzzy subset of X. Suppose $\lambda(x)=\alpha$ for $x \in X$. Then $\lambda$ can be expressed as union of all its fuzzy points, i.e, $\lambda=\vee_{x \in X} x_{\alpha}$. Here $\vee$ denote union. We will use the same notation $\vee$ to denote supremum of a set of numbers. Similarly $\wedge$ will be used to denote intersection of fuzzy sets as well as infimum of a set of real numbers.

Let $\lambda$ and $\mu$ be fuzzy subsets of $\mathrm{X}$, then we write $\lambda \subseteq \mu$ whenever $\lambda(x) \leq \mu(x)$. Let $\lambda$ be a fuzzy subset of a group $(G,+)$. Then we define a fuzzy subset $-\lambda$ as $-\lambda(x)=\lambda(-x)$. If $f$ is a function from $\mathrm{X}$ into $\mathrm{Y}$ and $\mu \in I^{Y}$, then $f^{-1}(\mu)$ is the fuzzy set in X defined by $f^{-1}(\mu)(x)=\mu(f(x))$. Equivalently, $f^{-1}(-\mu)=\mu \circ f$. Also, for $\rho \in I^{X}, f(\rho)$, is the member of $I^{Y}$ which is defined by

$$
f(\rho)(y)=\left\{\begin{array}{cc}
\sup \left\{\rho(x): x \in f^{-1}[y]\right\} & \text { if } f^{-1}[y] \text { is not empty } \\
0 & \text { otherwise }
\end{array}\right.
$$


For the definition of a fuzzy topology, we will use the one given by Lowen (1976) since his definition is more appropriate in our case. So, throughout this paper, by a fuzzy topology on a set $\mathrm{X}$ we will mean a sub-collection $\tau$ of $I^{X}$ satisfying the following conditions:

(i) $\tau$ contains every constant fuzzy subset in $\mathrm{X}$;

(ii) If $\mu_{1}, \mu_{2} \in \tau$, then $\mu_{1} \wedge \mu_{2} \in \tau$;

(iii) if $\mu_{i} \in \tau$ for each $i \in A$, then $\vee_{i \in A} \mu_{i} \in \tau$.

A fuzzy topological space is a set $\mathrm{X}$ on which there is given a fuzzy topology $\tau$. The elements of $\tau$ are the open fuzzy sets in X. Complement of an open fuzzy set is called a closed fuzzy set. Interior of a fuzzy set $\lambda$ is the union of all the open fuzzy set contained in $\lambda$ and the closure of $\lambda$ is the intersection of all fuzzy set containing $\lambda$. The interior and closure of $\lambda$ will be denoted by $\lambda^{o}$ and cl $\lambda$ respectively. A map f from a fuzzy topological space $\mathrm{X}$ to a fuzzy topological space $\mathrm{Y}$, is called continuous if $f^{-1}(\mu)$ is open in $\mathrm{X}$ for each open fuzzy set $\mu$ in $\mathrm{Y}$. Let $\mathrm{X}$ be a fuzzy topological space and $x \in X$. A fuzzy set $\mu$ in $\mathrm{X}$ is called a neighborhood of the fuzzy point $x_{\alpha}$ if there exists an open fuzzy set $\rho$ with $\rho \subseteq \mu$ and $x_{\alpha} \in \rho \subseteq \mu$. Given a crisp topological space $(X, T)$, the collection $\varpi(T)$, of all fuzzy sets in $\mathrm{X}$ which are lower semicontinuous, as functions from $\mathrm{X}$ to the unit interval $I=[0,1]$ equipped with the usual topology, is a fuzzy topology on X (Lowen, 1976). We will refer to the fuzzy topology $\varpi(T)$ as the fuzzy topology generated by the usual topology T. If $\left(X, T_{j}\right)_{j \in J}$ is a family of crisp topological spaces and T the product topology on $X=\prod_{j \in J} X_{j}$, then $\varpi(T)$ is the product of the fuzzy topologies $\varpi\left(T_{j}\right), j \in J$ (Lowen, 1977).

Result 2.1. (A. K. Katsaras, 1981) Let $\left(X_{i}, T_{i}\right), i=1,2,3$, be crisp topological spaces, $X=X_{1} \times X_{2}$, T the product of the topologies $T_{1}, T_{2}$ and $f:(X, T) \rightarrow\left(X_{3}, T_{3}\right)$ a continuous map. If $\delta$ is the product of the fuzzy topologies $\varpi\left(T_{1}\right)$ and $\varpi\left(T_{2}\right)$, then

$$
f:(X, \delta) \rightarrow\left(X_{3}, \varpi\left(T_{3}\right)\right)
$$

is fuzzy continuous.

Proof. Let $\mu \in \varpi\left(\tau_{3}\right)$. Then $\mu$ is a lower semicontinuous function from $\left(X_{3}, T_{3}\right)$ to the unit interval I. Since $\mathrm{f}$ is continuous with respect to the topologies $\mathrm{T}$ and $T_{3}$, it follows that the function $f^{-1}(\mu)=\mu \circ f$ is a lower semicontinuous function from (X, T) to the unit interval. Thus $f^{-1}(\mu) \in \varpi(T)=\delta$. This completes the proof.

Result 2.2. Let ( $\pi, \mathrm{G}, \mathrm{X}$ ) be a classical topological transformation group. If we equip $\mathrm{G}$ and $\mathrm{X}$ with the induced fuzzy topologies and $G \times X$, with the corresponding product fuzzy topology, then the mapping $\pi: G \times X \rightarrow X$ is fuzzy continuous.

Proof. It follows from the previous result.

Definition 2.3(Liu Ying-Ming, 1997) : Let $(X, \delta)$ be a fuzzy topological space and $Y \subseteq X$. Then the collection $\delta /_{Y}=\left\{\sigma /_{Y}: \sigma \in \delta\right\}$ is a fuzzy topology on Y. Then $(Y, \delta / Y)$ is called fuzzy subspace of $(\mathrm{X}, \delta)$.

Result 2.4 : Let $(\mathrm{X}, \delta)$ and $(\mathrm{Y}, \mu)$ be two fuzzy topological spaces. If $f: X \rightarrow Y$ is fuzzy continuous, then for any subset $A \subseteq X, f /{ }_{A}$ is fuzzy continuous. In particular an inclusion map is fuzzy continuous.

Result 2.5 (Liu Ying-Ming, 1997) Let $(\mathrm{X}, \delta),(\mathrm{Y}, \tau)$ and $(\mathrm{Z}, \kappa)$ be fuzzy topological spaces and $\mathrm{f}: \mathrm{X} \rightarrow \mathrm{Y}$ and $\mathrm{g}$ $: \mathrm{Y} \rightarrow \mathrm{Z}$ be any mappings. Then $\mathrm{f}, \mathrm{g}$ are fuzzy continuous $\Rightarrow$ gof is fuzzy continuous.

Definition 2.6 If $\sigma$ is a fuzzy subset of $\mathrm{X}$ and $\eta$ is a fuzzy subset of $\mathrm{Y}$, then the fuzzy subset $\sigma \times \eta$ on $X \times Y$ is defined as $(\sigma \times \eta)(x, y)=\min \{\sigma(x), \eta(y)\}$.

Definition 2.7 (Liu Ying-Ming, 1997) Let $(\mathrm{X}, \delta)$ and $(\mathrm{Y}, \tau)$ be two fuzzy topological spaces. Then $\mathrm{f}: \mathrm{X} \rightarrow \mathrm{Y}$ is fuzzy open (closed) if the image of every fuzzy open(closed) subset of $\mathrm{X}$ is fuzzy open(closed) in $\mathrm{Y}$.

Definition 2.8 (R. Lowen, 1976) Let X be a fuzzy topological space and $\lambda$ a fuzzy subset of X. An open fuzzy cover of $\lambda$ is a collection $\left\{\lambda_{\alpha}\right\}$ of open fuzzy subsets of $X$ such that $\lambda \subseteq \vee \lambda_{\alpha}$. If every open cover of $\lambda$ and $\varepsilon>0$ there exists a finite sub-collection $\left\{\lambda_{i}: i=1,2 \cdots n\right\}$ such that $\vee\left\{\lambda_{i}: i=1,2 \cdots n\right\} \geq \lambda-\varepsilon$ then $\lambda$ said to be fuzzy compact.

Result 2.9 (R. Lowen, 1976) (X, $\delta$ ) and (Y, $\tau)$ be two fuzzy topological spaces and $f: X \rightarrow Y$ be a fuzzy continuous function. If $\lambda$ is fuzzy compact subset of $\mathrm{X}$, then $f(\lambda)$ is fuzzy compact in $\mathrm{Y}$. 
Result 2.10 (R. Lowen, 1977). Let (X, $\delta$ ) and (Y, $\tau)$ be two fuzzy topological spaces and $\lambda, \mu$ are fuzzy compact subsets of $\mathrm{X}$ and $\mathrm{Y}$ respectively, then $\lambda \times \mu$ is fuzzy compact in $X \times Y$.

Definition 2.11 (Rajesh Kumar, 1993). Let $(\mathrm{G},+)$ be a group. Then a fuzzy subset $\lambda$ is said to be a fuzzy subgroup of $\mathrm{G}$ if $\lambda(x+y) \geq \min \{\lambda(x), \lambda(y))$ and $\lambda(-x)=\lambda(x)$

Remark : If $\lambda$ is a fuzzy subgroup of $\mathrm{G}$ then supp is a crisp subgroup of $\mathrm{G}$.

Definition 2.12 (N. Palaniappan, 2005) A fuzzy topological space $(X, \tau)$ is said to be product related to another fuzzy topological space (Y, $\delta$ ) if for any fuzzy set $v$ of $\mathrm{X}$ and $\zeta$ of $\mathrm{Y}$ whenever $\lambda^{c} \nsucceq v$ and $\mu^{c} \nsupseteq \zeta$ implies $\left(\lambda^{c} \times 1\right) \vee\left(1 \times \mu^{c}\right) \geq v \times \zeta$, where $\lambda \in \tau$ and $\mu \in \delta$, then there exist $\lambda_{1} \in \tau$ and $\mu_{1} \in \delta$ such that $\lambda_{1}^{c} \geq v$ or $\mu_{1}^{c} \geq \zeta$ and $\left(\lambda^{c} \times 1\right) \vee\left(1 \times \mu^{c}\right)=\left(\lambda_{1}^{c} \times 1\right) \vee\left(1 \times \mu_{1}^{c}\right)$.

Result 2.13. (N. Palaniappan, 2005) Let $(\mathrm{X}, \tau)$ be product related to $(\mathrm{Y}, \delta)$. Then for any fuzzy subset $\lambda$ of $\mathrm{X}$ and a fuzzy subset $\mu$ of $\mathrm{Y}, \operatorname{cl}(\lambda \times \mu)=c l \lambda \times c l \mu$.

\section{Fuzzy topological transformation groups}

In this section we will introduce the concept of fuzzy topological transformation group and prove some properties.

Definition 3.1 Let $\mathrm{X}$ be fuzzy topological space, $\mathrm{G}$ be a fuzzy topological group. If $\pi: G \times X \rightarrow X$ satisfies

(FTG1) $\pi(0, x)=x$

(FTG2) $\pi(s, \pi(t, x))=\pi(s+t, x)$

(FTG3) $\pi$ is fuzzy continuous

then $(\pi, G, X$, ) is called a fuzzy topological transformation group.

Definition 3.2 Let $t \in G$, then the t-transition of $\left(\pi, G, X\right.$, ) denoted by $\pi^{t}$ is the mapping : $\pi^{t}: X \rightarrow X$ such that $\pi^{t}(x)=\pi(t, x)$.

Result 3.3 (i) $\pi^{0}$ is the identity mapping of $\mathrm{X}$.

(ii) $\pi^{s} \pi^{t}=\pi^{s+t}$ for $s, t . \in G$.

(iii) $\pi^{t}$ is one-to-one mapping of $\mathrm{X}$ onto $\mathrm{X}$ and $-\left(\pi^{t}\right)=\pi^{-t}$.

(iv) For $t \in G, \pi^{t}$ is a fuzzy homomorphism of X onto X.

Proof. Straightforward.

Definition 3.4 The transition group of $(\pi, \mathrm{G}, \mathrm{X}$,$) is the set \mathrm{G}=\left\{\pi^{t}: t \in G\right\}$. The transition projection of (G, $\mathrm{X}$, $\pi)$ is the mapping $\theta: G \rightarrow G$ defined as $\theta(t)=\pi^{t}$.

Definition 3.5 (G, X, $\pi$ ) is said to be effective if $t \in G$ with $t \neq 0 \Rightarrow \pi^{t}(x) \neq x$ for some $\mathrm{x}$.

Result 3.6 (i) $G$ is a group of fuzzy homeomorphisms of $X$ onto $X$

(ii) $\theta$ is a group homomorphism of $\mathrm{G}$ onto $\mathrm{G}$.

(iii) $\theta$ is one-one iff $(\pi, \mathrm{G}, \mathrm{X})$ is effective.

Proof. Straightforward.

Definition 3.7 Let $x \in X$, then the $\mathrm{x}$-motion of $(\pi, \mathrm{G}, \mathrm{X})$ is the mapping $\pi_{x}: G \rightarrow X$ such that $\pi_{x}(t)=\pi(t, x)$.

Result 3.8 $\pi_{x}$ is a fuzzy continuous mapping of $\mathrm{G}$ into $\mathrm{X}$.

Proof. Straightforward.

Result 3.9 Let X, Y, Z be fuzzy topological spaces and f : $X \times Y \rightarrow Z$ be a fuzzy continuous map. If $a_{\alpha}, b_{\beta}$ be fuzzy points of $\mathrm{X}$ and $\mathrm{Y}$ respectively and $\gamma$ be a fuzzy neighbourhood of $f\left(a_{\alpha}, b_{\beta}\right)$ then there exists fuzzy neighbourhoods $\eta$ and $\rho$ of $a_{\alpha}$ and $b_{\beta}$ respectively such that $f(\eta \times \rho) \subseteq \gamma$.

Proof. Without loss of generality we can assume that $\gamma$ is fuzzy open. As $\mathrm{f}$ is continuous $f^{-1}(\gamma)$ is a fuzzy open set containing $a_{\alpha} \times b_{\beta}$. So there exists basic fuzzy open sets say, $\eta$ of $a_{\alpha}$ and $\rho$ of $b_{\beta}$ such that $a_{\alpha} \times b_{\beta} \in \eta \times \rho \subseteq$ $f^{-1}(\gamma)$. Which gives $f\left(a_{\alpha} \times b_{\beta}\right) \in f(\eta \times \rho) \subseteq \gamma$. 
Result 3.10 Let X, Y, Z be fuzzy topological spaces and $\mathrm{f}: X \times Y \rightarrow Z$ be a fuzzy continuous map. If $\lambda$ and $\mu$ are fuzzy compact subsets of $\mathrm{X}$ and $\mathrm{Y}$ respectively and $\gamma$ is a fuzzy neighbourhood of $f(\lambda \times \mu)$, then for any $\varepsilon>0$, there exists fuzzy open sets $\lambda^{\prime}$ and $\mu^{\prime}$ such that $\lambda^{\prime} \geq \lambda-\varepsilon$ and $\mu^{\prime} \geq \mu-\varepsilon$ and $f\left(\lambda^{\prime} \times \mu^{\prime}\right) \subseteq \gamma$.

Proof. Let $\varepsilon>0$ be arbitrary. Let $x \in X$ be arbitrarily fixed and suppose $\lambda(x)=\alpha$. Then for any $y \in Y$ with $\mu(y)=\beta$, by previous result there exist fuzzy open sets $\lambda_{y} \ni x_{\alpha}$ and $\mu_{y} \ni y_{\beta}$ such that $f\left(x_{\alpha} \times y_{\beta}\right) \in f\left(\lambda_{y} \times \mu_{y}\right) \subseteq \gamma$. This is true for each $y \in Y$. Thus the collection $C_{\mu}=\left\{\mu_{y}: y \in Y\right\}$ is an open cover of $\mu$. As $\mu$ is compact there is a finite sub-collection say $S_{\mu}$ of $C_{\mu}$ satisfying $\vee\left\{\mu_{y}: \mu_{y} \in S\right\} \geq \mu-\varepsilon$. Let $\mu_{x}$ denote the union of all members of $S_{\mu}$ and $\lambda_{x}$ denote the intersection of the corresponding $\lambda_{y}^{\prime} s$. Then $\lambda_{x}$ is a fuzzy open set containing $x_{\alpha}$ and $\mu_{x}$ is a fuzzy open set satisfying $\mu_{x} \geq \mu-\varepsilon$.

But this is true for each $x \in X$. Thus we get a collection $\left\{\mu_{x}: x \in X\right\}$ of fuzzy open sets each satisfying $\mu_{x} \geq \mu-\varepsilon$ and another collection $\left\{\lambda_{x}: x \in X\right\}$ of fuzzy open sets such that $x_{\alpha} \in \lambda_{x}(\alpha=\lambda(x))$. Then $C_{\lambda}=\left\{\lambda_{x}: x \in X\right\}$ is a cover of $\lambda$. As $\lambda$ is compact there exists a finite collection $S_{\lambda}$ of $C_{\lambda}$ satisfying $\vee\left\{\lambda_{x}: \lambda_{x} \in S_{\lambda}\right\} \geq \lambda-\varepsilon$. Let $\lambda^{\prime}$ denote the union of the members of $S_{\lambda}$ and $\mu^{\prime}$ denote the intersection of the corresponding $\mu_{x}$. Then $\lambda^{\prime}$ is a fuzzy open set satisfying $\lambda^{\prime} \geq \lambda-\varepsilon$ and $\mu^{\prime}$ is a fuzzy open set satisfying $\mu^{\prime} \geq \mu-\varepsilon$. Further then $f\left(\lambda^{\prime} \times \mu^{\prime}\right) \subseteq \gamma$.

\section{Result 3.11}

(i) For $t \in G$ and a fuzzy subset $\mu$ of $\mathrm{X}, c l \pi(t \times \mu)=\pi(t \times c l \mu)$

(ii) Let $\mathrm{G}$ and $\mathrm{X}$ be product related, then for a fuzzy subset $\lambda$ of $\mathrm{G}$ and a fuzzy subset $\mu$ of $\mathrm{X}, \pi(c l \lambda \times c l \mu) \subseteq$ $c l \pi(\lambda \times \mu)$ and $c l \pi(c l \lambda \times \mu)=c l \pi(\lambda \times c l \mu)=c l \pi(\lambda \times \mu)$.

(iii) If $\lambda$ is a compact fuzzy subset of $\mathrm{G}$ and $\mu$ is a compact fuzzy subset of $\mathrm{X}$, then $\pi(\lambda \times \mu)$ is a compact fuzzy subset of $X$.

(iv) If $\lambda$ is a compact fuzzy subset of $\mathrm{G}$ and $\mu$ is a compact fuzzy subset of $\mathrm{X}$, and $\gamma$ is a fuzzy neighbourhood of $\pi(\lambda \times \mu)$, then for any $\varepsilon>0$, there exists fuzzy open sets $\lambda^{\prime}$ and $\mu^{\prime}$ such that $\lambda^{\prime} \geq \lambda-\varepsilon$ and $\mu^{\prime} \geq \mu-\varepsilon$ such that $f\left(\lambda^{\prime} \times \mu^{\prime}\right) \subseteq \gamma$.

(v) $\pi^{t} \mu=\mu \pi^{-t}$ for any $t \in G$.

(vi) $\pi^{t} \mu^{c}=1-\pi^{t} \mu$

Proof. (i) Since $\pi^{t}$ is a homeomorphism $c l \pi^{t}(\mu)=\pi^{t}(c l \mu)$, i.e., $c l \pi(t \times \mu)=\pi(t \times c l \mu)$

(ii) Since $\mathrm{G}$ and $\mathrm{X}$ are product related, $(\operatorname{cl} \times \times \operatorname{cl} \mu)=\operatorname{cl}(\lambda \times \mu)$ which implies

$$
\pi(\operatorname{cl} \lambda \times \operatorname{cl} \mu)=\pi\{c l(\lambda \times \mu)\}
$$

$\Rightarrow \pi(c l \lambda \times c l \mu)=\pi\{c l(\lambda \times \mu)\} \subseteq c l \pi(\lambda \times \mu)$, since $\pi$ is continuous.

Again $\pi(\lambda \times \mu) \subseteq \pi(c l \lambda \times \mu) \subseteq \pi(\operatorname{cl} \times \operatorname{cl} \mu) \subseteq \operatorname{cl} \pi(\lambda \times \mu)$

and $\pi(\lambda \times \mu) \subseteq \pi(\lambda \times c l \mu) \subseteq \pi(c l \lambda \times c l \mu) \subseteq c l \pi(\lambda \times \mu)$ and consequently $c l \pi(c l \lambda \times \mu)=c l \pi(\lambda \times c l \mu)=c l \pi(\lambda \times \mu)$.

(iii) $\lambda$ and $\mu$ are fuzzy compact, so $\lambda \times \mu$ is fuzzy compact. As continuous image of a fuzzy compact set is fuzzy compact $\pi(\lambda \times \mu)$ is fuzzy compact.

(iv) Follows from Result 3.10.

(v) We have for any $u \in X, \pi^{t}(\mu)(u)=\pi(t \times \mu)(u)=\sup \{(t \times \mu)(s, x): \pi(s, x)=u\}$

$=\sup \{(t(s) \wedge \mu(x): \pi(s, x)=u\}$

$=\sup \{(t(t) \wedge \mu(x): \pi(t, x)=u\}$, since $t(s) \neq 0$ only when $t=s$.

$=\mu(x)$ where $\pi(s, x)=u$

$=\mu\left(\pi^{-t}\right)(u)$.

(vi) From (v) we have $\pi^{t}(\mu)=\mu\left(\pi^{-t}\right)$ for any $\mu \in I^{X}$ and $t \in G$.

Now for any $x \in X$, we have $\left(\pi^{t} \mu^{c}\right)(x)=\left(\mu^{c} \pi^{-t}\right)(x)=\mu^{c}\left(\left(\pi^{-t}\right)(x)\right)=1-\left(\mu \pi^{-t}\right)(x)$

$=\left(1-\mu \pi^{-t}\right)(x)$. Therefore $\pi^{t} \mu^{c}=1-\mu \pi^{-t}$. 
Result 3.12. Let $\alpha$ be a constant fuzzy subset of $\mathrm{G}$ and $\mu \in I^{X}$ be fuzzy open. Then $\pi(\alpha \times \mu)$ is fuzzy open.

Proof. We have for any $u \in X, \pi(\alpha \times \mu)(u)=\sup \{(\alpha \times \mu)(t, x): \pi(t, x)=u\}$

$=\sup \{(\alpha(t) \wedge \mu(x): \pi(t, x)=u\}=\sup \{(\alpha \wedge \mu(x): \pi(t, x)=u\}$

$=\alpha \wedge \sup \left\{\mu(x): \pi^{t}(x)=u\right\}=\alpha \wedge \sup \left\{\mu\left(\pi^{-t}(u)\right): \pi^{-t}(u)=x\right\}$

$=\alpha \wedge \sup \left\{\pi^{t} \mu(u): \pi^{-t}(u)=x\right\}$, since $\mu \pi^{-t}=\pi^{t} \mu$.

$=\alpha \wedge\left\{\vee\left\{\pi^{t} \mu(u)\right\}\right.$ where $\pi^{-t}(u)=x$

$=\left\{\alpha \wedge\left\{\vee\left(\pi^{t} \mu\right)\right\}\right\}(u)$, where $\pi^{-t}(u)=x$

Thus $\pi(\alpha \times \mu)=\alpha \wedge\left\{\vee\left(\pi^{t} \mu\right)\right\}$. Now each $\pi^{t}$ is open and $\mu$ is open so $\pi^{t} \mu$ is open. Also by definition of fuzzy topology is open. Consequently $\alpha \wedge\left\{\vee\left(\pi^{t} \mu\right)\right\}$ is open. Hence $\pi(\alpha \times \mu)$ is open.

Corollary 3.13. Let $\mu$ be a fuzzy open subset of X. then for any fuzzy point $t_{\alpha}$ of G, $\pi\left(t_{\alpha} \times \mu\right)$ is fuzzy open.

Proof. We have for any $u \in X, \pi\left(t_{\alpha} \times \mu\right)(u)=\sup \left\{\left(t_{\alpha} \times \mu\right)(s, x): \pi(s, x)=u\right\}$

$=\sup \left\{t_{\alpha}(s) \wedge \mu(x): \pi(s, x)=u\right\}=\alpha \wedge \mu(x): \pi(t, x)=u$

$=\alpha \wedge \mu(x): \pi^{t}(x)=u$

$=\alpha \wedge \mu\left(\pi^{-t}(u)\right)$

$=\alpha \wedge \pi^{t} \mu(u)$, since $\mu \pi^{-t}=\pi^{t} \mu$.

$=\left(\alpha \wedge \pi^{t} \mu\right)(u)$, considering $\alpha$ as a constant fuzzy subset on X.

Thus $\pi(\alpha \times \mu)=\alpha \wedge \pi^{t} \mu$. Now $\pi^{t}$ is open and $\mu$ is open so $\pi^{t} \mu$ is open. Also by definition of fuzzy topology $\alpha$ is open. Consequently $\alpha \wedge \pi^{t}$ is fuzzy open. Hence $\pi\left(t_{\alpha} \times \mu\right)$ is open.

Corollary 3.14. Let $\lambda$ be any fuzzy subset of $\mathrm{G}$ and $\mu \in I^{X}$ be fuzzy open, then $\pi(\lambda \times \mu)$ is fuzzy open.

Proof. We have $\lambda=\vee t_{\alpha}$, where $\alpha=\lambda(x)$. So $\pi(\lambda \times \mu)=\pi\left(\vee t_{\alpha} \times \mu\right)=\vee \pi\left(t_{\alpha} \times \mu\right)$. As already proved each $\pi\left(t_{\alpha}, \times \mu\right)$ is open and hence $\pi(\lambda \times \mu)$ is open.

Result 3.15 Let $\mu$ be a fuzzy closed subset of X. then for any fuzzy point $t_{\alpha}$ of G, $\pi\left(t_{\alpha} \times \mu\right)$ is fuzzy closed.

Proof. We have for any $u \in X, \pi\left(t_{\alpha} \times \mu\right)(u)=\sup \left\{\left(t_{\alpha} \mu\right)(s, x): \pi(s, x)=u\right\}$

$=\sup \left\{t_{\alpha}(s) \wedge \mu(x): \pi(s, x)=u\right\}$

$=\alpha \wedge \mu(x): \pi(t, x)=u$, since $t_{\alpha}(s) \neq 0$ only when $s=t$.

$=\alpha \wedge \mu(x): \pi^{t}(x)=u$

$=\alpha \wedge \mu\left(\pi^{-t}(u)\right)$

$=\alpha \wedge \pi^{t} \mu(u)$, since $\mu \pi^{-t}=\pi^{t} \mu$.

$=\left(\alpha \wedge \pi^{t} \mu\right)(u)$, considering $\alpha$ as a constant fuzzy subset on X.

Thus $\pi(\alpha \times \mu)=\alpha \wedge \pi^{t} \mu$. Now $\pi^{t}$ is closed and $\mu$ is closed so $\pi^{t} \mu$ is closed. Also by definition of fuzzy topology is closed. Consequently $\alpha \wedge \pi^{t} \mu$ is fuzzy closed. Hence $\pi\left(t_{\alpha} \times \mu\right)$ is closed.

Corollary 3.16. Let $\lambda$ be any fuzzy subset of $\mathrm{G}$ and $\mu \in I^{X}$ be fuzzy closed. If supp $\lambda$ is finite, then $\pi(\lambda \times \mu)$ is fuzzy closed.

Proof. We have $\lambda=\vee t_{\alpha}$, where $\alpha=\lambda(x)$. So $\pi(\lambda \times \mu)=\pi\left(\vee t_{\alpha} \times \mu\right)=\vee \pi\left(t_{\alpha} \times \mu\right)$. As already proved each $\pi\left(t_{\alpha}, \times \mu\right)$ is closed. Also since supp $\lambda$ is finite, the union is over finite number of closed fuzzy subsets. Hence $\pi(\lambda \times \mu)$ is closed.

\section{Invariant fuzzy subsets}

In this section we will introduce the notion of invariance of a fuzzy subset of $\mathrm{X}$ under the action of a fuzzy subset of G.

Definition 4.1 Let $\lambda$ a fuzzy subset of $\mathrm{G}$ and $\mu$ a fuzzy subset of $\mathrm{X}$. Then $\mu$ is said to be invariant under $\lambda$ or $\lambda$-invariant provided that $\pi(\lambda \times \mu) \subseteq \mu$. If $\lambda=\chi_{G}$ then $\mu$ is simply said to be invariant. If $\lambda=\chi_{G}$ and $\mu$ is a crisp 
subset, then fuzzy invariance reduces to crisp invariance.

Result 4.2 (i) If $\mu$ is a fuzzy subset of $X$, and $\lambda$ is a fuzzy subgroup of $X$ satisfying $\lambda(0)=1$, then the following statements are pairwise equivalent : $\pi(\lambda \times \mu) \subseteq \mu$.; $\pi(\lambda \times \mu)=\mu ; \pi(\lambda(t) \times \mu) \subseteq \mu \forall t \in G$.

(ii) 0 and 1 are invariant.

(iii) If $\pi(t \times \mu) \subseteq \mu$, then $\pi\left(-t \times \mu^{c}\right) \subseteq \mu^{c}$ and conversely

(iv) If $\mu$ is $\lambda$-invariant then int $\mu$ is $\lambda$-invariant and $\operatorname{cl} \mu$ is $\lambda$-invariant provided $\mathrm{G}$ and $\mathrm{X}$ are product related.

(v) If $\left\{\mu_{i}\right\}$ is a collection of $\lambda$-invariant fuzzy subset, then $\vee \mu_{i}$ and $\wedge \mu_{i}$ are $\lambda$-invariant.

(vi) Let $\mathrm{A}$ be a crisp subset of $\mathrm{G}$ and $\mu$ a fuzzy subset of $\mathrm{X}$., then $\mu$ is $\chi_{A}$-invariant iff $\mu^{c}$ is $\chi-A^{-}$invariant.

Proof (i) First we show $\pi(\lambda \times \mu) \subseteq \mu \Leftrightarrow \pi(\lambda \times \mu)=\mu$.

Suppose $\pi(\lambda \times \mu) \subseteq \mu$. We have for any $\mathrm{u}$ in $\mathrm{X}, \pi(\lambda \times \mu)(u)=\sup \{(\lambda \times \mu)(t, x): \pi(t, x)=u\}$

$=\sup \{(\lambda(t) \wedge \mu(x): \pi(t, x)=u\}$

$\geq \lambda(0) \wedge \mu(u)$, since $\pi(0, u)=u$

$=\mu(u)$ since $\lambda(0)=1$.

Thus $\pi(\lambda \times \mu)=\mu$. Consequently $\pi(\lambda \times \mu) \subseteq \mu$. $\Leftrightarrow \pi(\lambda \times \mu)=\mu$.

Next we show $\pi(\lambda(t) \times \mu) \subseteq \mu \Leftrightarrow \forall t \in G . \pi(\lambda \times \mu) \subseteq \mu$

Given $\pi\left(t_{\alpha} \times \mu\right) \subseteq \mu$ for all $t_{\alpha}: \alpha=\lambda(t)$.

Now $\pi(\lambda \times \mu)=\pi\left(\vee t_{\alpha} \times \mu\right)=\vee \pi\left(t_{\alpha} \times \mu\right) \subseteq \mu$

(ii) Trivial

(iii) We have $\pi(t \times \mu) \subseteq \mu \Rightarrow \pi^{t}(\mu) \subseteq \mu$

And $\pi\left(-t \times \mu^{c}\right)=\pi^{-t}\left(\mu^{c}\right)$

Now $1-\mu \subseteq 1-\pi^{t}(\mu)=\mu^{c} \pi^{-t} \Rightarrow \mu^{c} \subseteq \pi^{t} \mu^{c} \Rightarrow \pi^{-t} \mu^{c} \subseteq \mu^{c} \Rightarrow \pi\left(-t \times \mu^{c}\right) \subseteq \mu^{c}$

(iv) We have $\pi(\lambda \times \mu) \subseteq \mu$. Now $\pi\left(\lambda \times \mu^{o}\right) \subseteq \pi(\lambda \times \mu) \subseteq \mu$. Now $\mu^{o}$ is open so $\pi\left(\lambda \times \mu^{o}\right)$ is open and contained in $\mu$. But $\mu^{o}$ is the largest open fuzzy set contained in $\mu$.

Hence $\pi\left(\lambda \times \mu^{o}\right) \subseteq \mu^{o}$.

Since $\mathrm{G}$ and $\mathrm{X}$ are product related $\lambda \times \operatorname{cl} \mu \subseteq \operatorname{cl} \times \times \operatorname{cl} \mu=\operatorname{cl}(\lambda \times \mu)$

$\Rightarrow \pi(\lambda \times \operatorname{cl} \mu) \subseteq \pi\{c l(\lambda \times \mu)\} \subseteq \operatorname{cl}(\lambda \times \mu)$, since $\pi$ is continuous

$\subseteq c l \mu$.

(v) We have $\pi\left(\lambda \times \vee \mu_{i}\right)=\pi\left\{\vee\left(\lambda \times \mu_{i}\right)\right\}=\vee \pi\left(\lambda \times \mu_{i}\right) \subseteq \mu$.

Similarly $\pi\left(\lambda \times \wedge \mu_{i}\right)=\pi\left\{\wedge\left(\lambda \times \mu_{i}\right)\right\} \subseteq \wedge \pi\left(\lambda \times \mu_{i}\right) \subseteq \mu$.

(vi) It is sufficient to show that $\pi(t \times \mu) \subseteq \mu$, then $\pi\left(-t \times \mu^{c}\right) \subseteq \mu^{c}$ and conversely for $t \in A$. Hence it follows from (iii)

\section{Fuzzy Orbits}

In this section we will introduce the notion of orbits in fuzzy setting and extend some classical results.

Definition 5.1 Let $x \in X$ and $\lambda$ a fuzzy subgroup of G. Then the fuzzy orbit of $\mathrm{x}$ under $\lambda$ or the $\lambda$ - orbit of $\mathrm{x}$ is defined to be fuzzy subset $\pi(\lambda \times x)$. The fuzzy orbit closure of $\mathrm{x}$ under $\lambda$ or the $\lambda$ - orbit closure of $\mathrm{x}$ is defined to be the fuzzy subset $c l \pi(\lambda \times x)$.

When $\lambda=\chi_{G}$, then the fuzzy orbit coincides with the crisp orbit. We will denote the orbit of $\mathrm{x}$ under $\lambda$ by $\lambda_{x}$. We assume that $\mathrm{G}$ and $\mathrm{X}$ are product related and that $\lambda(0)=1$.

Remark : $\lambda_{x}(u)=\sup \{\lambda(t): \pi(t, x)=u\}$.

We have $\lambda_{x}(u)=\pi(\lambda, x)(u)=\sup \{(\lambda, x)(t, y):(t, y)=u\}$ 
$=\sup \{(\lambda(t) \wedge x(y): \pi(t, y)=u\}$

$=\sup \{(\lambda(t): \pi(t, x)=u\}$, since $x(y) \neq 0$ iff $x=y$.

Result 5.2 Let $x, y \in X$, then

(i) $\lambda_{x}(y)=\lambda_{y}(x)$

Proof. We have $\lambda_{x}(y)=\sup \{\lambda(t): \pi(t, x)=y\}=\sup \{\lambda(t): \pi(-t, y)=x\}$

$=\sup \{\lambda(-t): \pi(-t, y)=x\}=\lambda_{y}(x)$.

(ii) $\lambda$-orbit of $\mathrm{x}$ is $\lambda$-invariant.

Proof. We have for any $u \in X$,

$\pi\left(\lambda \times \lambda_{x}\right)(u)=\sup \left\{\left(\lambda \times \lambda_{x}\right)(t, y): \pi(t, y)=u\right\}=\sup \left\{\lambda(t) \wedge \lambda_{x}(y): \pi(t, y)=u\right\}$.

$=\sup [\lambda(t) \wedge \sup \{\lambda(s): \pi(s, x)=y\}: \pi(t, y)=u\}]$

$\left.=\sup _{t} \sup _{s}\{\lambda(t) \wedge \lambda(s): \pi(t+s, x)=u\}\right\}$

$\leq \sup _{t} \sup _{s}\{\lambda(t+s): \pi(t+s, x)=u\}$, since $\lambda$ is a fuzzy subgroup of $\mathrm{G}$

$=\sup \{\lambda(r): \pi(r, x)=u\}=\lambda_{x}(u)$. Hence $\pi\left(\lambda \times \lambda_{x}\right) \subseteq \lambda_{x}$.

(iii) Let $\eta \in I^{G}$ be $\lambda$-invariant and $\eta(x)=1$, then $\lambda_{x} \subseteq \eta$. In other words fuzzy orbit of $\mathrm{x}$ is the least $\lambda$-invariant fuzzy subset containing the fuzzy point $\mathrm{x}$.

Proof. We have for any $u \in X$,

$\lambda_{x}(u)=\sup \{\lambda(t): \pi(t, x)=u\}=\sup \{\lambda(t) \wedge \eta(x): \pi(t, x)=u\}$, since $\eta(x)=1$.

$=\pi(\lambda \times \eta)(u) \subseteq \eta(u)$.

(iv) The closure of $\lambda$-orbit of $\mathrm{x}$ is $\lambda$-invariant.

Proof. Since $\mathrm{G}$ and $\mathrm{X}$ are product related, we have $\pi\left(\lambda \times \operatorname{cl} \lambda_{x}\right) \subseteq \pi\left(\operatorname{cl}\left(\lambda \times \lambda_{x}\right)\right)$

$\subseteq c l \pi\left(\lambda \times \lambda_{x}\right)$, since $\pi$ is continuous

$\subseteq \operatorname{cl} \lambda_{x}$, since $\pi\left(\lambda \times \lambda_{x}\right) \subseteq \lambda_{x}$.

(v) Let $\eta \in I^{G}$ be closed, $\lambda$-invariant and $\eta(x)=1$, then $c l \lambda_{x} \subseteq \eta$. In other words fuzzy orbit of $\mathrm{x}$ is the least $\lambda$-invariant fuzzy closed subset containing the fuzzy point $\mathrm{x}$.

Proof. Since $\lambda_{x}$ is $\lambda$-invariant, $\pi\left(\lambda \times \lambda_{x}\right)=\lambda_{x}$ by Result 2(i). So $\lambda_{x}=\pi(\lambda \times x) \subseteq \pi(\lambda \times \eta)$, since $\eta(x)=1$,

$\subseteq \eta \Rightarrow \lambda_{x} \subseteq \eta \Rightarrow c l \lambda_{x} \subseteq c l \eta \Rightarrow c l \lambda_{x} \subseteq \eta$, since $\eta$ is closed.

(vi) If $c l \lambda_{x}(y)=1$, then $c l \lambda_{y} \subseteq c l \lambda_{x}$.

Proof. Since $c l \lambda_{y}$ is the least closed $\lambda$-invariant fuzzy subset containing $y$, the result follows.

(vii) The collection $\left\{\lambda_{x}: x \in X\right\}$ is a cover of 1 .

Proof. This is because for each $x \in X, \lambda_{x}(x)=1$.

(viii) Let $\mathrm{x}, \mathrm{y} \in \mathrm{X}$ such that $\lambda_{x}(y)>0$. Then, $\lambda_{x}(u)>0 \Leftrightarrow \lambda_{y}(u)>0$ for any $\mathrm{u}$ in $\mathrm{X}$.

Proof. Given $\lambda_{x}(y)>0$. So $\exists t \in G$ with $\lambda(t)>0: \pi(t, x)=y$. This implies $\pi(-t, y)=x$. Let $\lambda_{x}(u)>0$, then there exist $s \in G$ with $\lambda(s)>0: \pi(s, x)=u$.

So $\pi(s-t, y)=u$ and $\lambda(s-t) \geq \lambda(s) \wedge \lambda(-t)=\lambda(s) \wedge \lambda(t)>0$, since $\lambda$ is a fuzzy subgroup $\Rightarrow \lambda_{y}(u)>0$.

Similarly $\lambda_{y}(u)>0 \Rightarrow \lambda_{x}(u)$.

(ix) If $\lambda_{x}(y)=0$, then $\lambda_{x}(u)=0$, for all $\mathrm{u}$ in $\mathrm{X}$ such that $\lambda_{y}(u)>0$

Proof. We have $\lambda_{x}(y)=0$, so there exists no $t \in G$ with $\lambda(t)>0: \pi(t, x)=y--(i)$ Let $u \in X$ such that $\lambda_{y}(u)>0 \Rightarrow \exists r \in G$ with $\lambda(r)>0: \pi(r, y)=u$. $--(i i)$

Suppose $\lambda_{x}(u)>0$, then $\exists s \in G$ with $\lambda(s)>0: \pi(s, x)=u \Rightarrow \pi(-r,(s, x))=\pi(-r, u)=y$ using (ii)

$\Rightarrow \pi(-r+s, x))=y$, where $\lambda(-r+s) \geq \lambda(-r) \wedge \lambda(s)=\lambda(r) \wedge \lambda(s)>0$, since $\lambda$ is a fuzzy subgroup But this 
contradicts (i). Hence the result.

We consider the collection of all $\lambda$-orbits and define a relation on it as $\lambda_{x} \sim \lambda_{y}$ if $\lambda_{x}(y)>0$. Then it can be easily verified that this relation is an equivalence relation, where the equivalence class of $\lambda_{x}$ is $\left[\lambda_{x}\right]=\left\{\lambda_{y}: \lambda_{x}(y)>0\right\}$. So $\left[\lambda_{x}\right]$ is a fuzzy subset of $\mathrm{X}$ defined as $\left[\lambda_{x}\right](y)=\lambda_{x}(y)$.

The collection $\left\{\operatorname{supp}\left[\lambda_{x}\right]: x \in X\right\}$ is a crisp partition of $\mathrm{X}$.

Let us denote the set of all equivalence classes by $X / \lambda$, i.e., $X / \lambda_{\lambda}=\left\{\left[\lambda_{x}\right]: x \in X\right\}$. Define a map $f: X \rightarrow X / \lambda$ given by $f(x)=\left[\lambda_{x}\right]$. We equip $X / \lambda$ with the corresponding quotient topology.

Result 5.3 The map $f: X \rightarrow X / \lambda$ given by $f(x)=\left[\lambda_{x}\right]$ is an open map.

Proof. Let $\mu$ be an open fuzzy subset of X. To show $f(\mu)$ is fuzzy open in $X /{ }_{\lambda}$. Since $X /{ }_{\lambda}$ has quotient topology with respect to $\mathrm{f}$, it is sufficient to show $f^{-1}\{f(\mu)\}$ is open in $\mathrm{X}$.

We have $f^{-1}\{f(\mu)\}(x)=f(\mu)(f(x))=f(\mu)\left[\lambda_{x}\right]=\sup \left\{\mu(y): f(y)=\left[\lambda_{x}\right]\right\}$

$=\sup \left\{\mu(y):\left[\lambda_{y}\right]=\left[\lambda_{x}\right]\right\}=\sup \left\{\mu(y): \lambda_{y} \in\left[\lambda_{x}\right]\right\}$

$=\sup \left\{\mu(y): \lambda_{x}(y)>0\right\}=\sup \{\mu(y): \pi(t, x)=y$ for some $t \in G$ with $\lambda(t)>0\}$

$=\sup \left\{\mu\left(\pi^{-t}(x)\right): \pi(t, x)=y\right.$ for some $t \in G$ with $\left.\lambda(t)>0\right\}$

$=\sup \left\{\pi^{t} \mu(x): \pi(t, x)=y\right.$ for some $t \in G$ with $\left.\lambda(t)>0\right\}$

$=\vee \pi^{t} \mu(x)$, where $t \in G$ with $\lambda(t)>0$

Hence $f^{-1}\{f(\mu)\}=\vee \pi^{t} \mu$.

Now each $\pi^{t}$ is a homeomorphism and $\mu$ is open and so $\pi^{t} \mu$ is open. Consequently $\vee \pi^{t} \mu$. is open. Hence $f^{-1}\{f(\mu)\}$ is open.

Consequently $\mathrm{f}$ is a fuzzy open map.

Corollary 5.4 : If $\lambda$ has finite support, then the map $f: X \rightarrow X / \lambda$ given by $f(x)=\lambda_{x}$ is a closed map.

Proof. Let $\mu$ be a closed fuzzy subset of $X$. To show $f(\mu)$ is fuzzy closed in $X / \lambda$. Since $X / \lambda$ has quotient topology with respect to $\mathrm{f}$, it is sufficient to show $f^{-1}\{f(\mu)\}$ is closed in $\mathrm{X}$.

We have $f^{-1}\{f(\mu)\}(x)=f(\mu)(f(x))=f(\mu)\left[\lambda_{x}\right]=\sup \left\{\mu(y): f(y)=\left[\lambda_{x}\right]\right\}$

$=\sup \left\{\mu(y):\left[\lambda_{y}\right]=\left[\lambda_{x}\right]\right\}=\sup \left\{\mu(y): \lambda_{y} \in\left[\lambda_{x}\right]\right\}$

$=\sup \left\{\mu(y): \lambda_{x}(y)>0\right\}=\sup \{\mu(y): \pi(t, x)=y$ for some $t \in G$ with $\lambda(t)>0\}$

$=\sup \left\{\mu\left(\pi^{-t}(x)\right): \pi(t, x)=y\right.$ for some $t \in G$ with $\left.\lambda(t)>0\right\}$

$=\sup \left\{\pi^{t} \mu(x): \pi(t, x)=y\right.$ for some $t \in G$ with $\left.\lambda(t)>0\right\}$

$=\vee \pi^{t} \mu(x)$, where $t \in G$ and $\lambda(t)>0$

Hence $f^{-1}\left\{f(\mu)=\vee \pi^{t} \mu\right.$. Now each $\pi^{t}$ is a homeomorphism and $\mu$ is closed and so each $\pi^{t} \mu$ is closed. As supp $\lambda$ is finite supremum is over finite number of $t^{\prime} s$.

Consequently $\vee \pi^{t} \mu$ being union of finite number of closed fuzzy sets is closed.

Hence $f^{-1}\{f(\mu)\}$ is closed.

Consequently $\mathrm{f}$ is a fuzzy closed map.

\section{Construction of new fuzzy topological transformation groups from given ones}

In this section we will construct new fuzzy topological transformation groups from given ones.

Result 5.1 Let $(\pi, G, X)$ and $(\varphi, \mathrm{G}, \mathrm{Y})$ be two fuzzy topological transformation groups. Define $\Psi: G \times(X \times Y) \rightarrow$ $X \times Y$ as $\Psi(t,(x, y))=(\pi(t, x),(t, y))$. Then $(\Psi, G,(X \times Y))$ is a fuzzy topological transformation group.

Proof. As $\pi$ is fuzzy continuous $\Psi$ is fuzzy continuous.

Also $\Psi(0,(\mathrm{x}, \mathrm{y}))=(\pi(0, \mathrm{x}), \pi(0, \mathrm{y}))=(\mathrm{x}, \mathrm{y})$ and

$\Psi(\mathrm{s}+\mathrm{t},(\mathrm{x}, \mathrm{y}))=(\pi(\mathrm{s}+\mathrm{t}, \mathrm{x}), \pi(\mathrm{s}+\mathrm{t}, \mathrm{y}))$ 
$=(\pi(\mathrm{s}, \pi(\mathrm{t}, \mathrm{x}), \pi(\mathrm{s}, \pi(\mathrm{t}, \mathrm{y}))=\Psi(\mathrm{s},(\pi(\mathrm{t}, \mathrm{x}), \pi(\mathrm{t}, \mathrm{y})))=\Psi(\mathrm{s}, \Psi(\mathrm{t},(\mathrm{x}, \mathrm{y}))$.

Thus (FGT1), (FGT2) and FGT3) are satisfied and hence $(\Psi, \mathrm{G},(\mathrm{X} \times \mathrm{Y}))$ is a fuzzy topological transformation group.

Result 5.2 Let $\lambda$ be a fuzzy subgroup of $\mathrm{G}$ and $\mu \in I^{X}$ such that $\pi(\lambda \times \mu) \subseteq \mu$. Then ( $\operatorname{supp} \lambda, \operatorname{supp} \mu, \pi / \operatorname{supp} \lambda \times \operatorname{supp} \mu$ ) is fuzzy topological transformation group.

Proof. Since $\lambda$ is fuzzy subgroup, supp $\lambda$ is an ordinary group Since subgroup of a fuzzy topological group is a fuzzy topological group, supp $\lambda$ is a fuzzy topological group. Since $\pi(\lambda \times \mu) \subseteq \mu$, range of $\pi /$ supp $\lambda \times$ supp $\mu$ ) is contained in $\operatorname{supp} \mu$. Also restriction of fuzzy continuous function if fuzzy continuous. Hence the result.

Corollary 5.3 Let $\lambda$ be a fuzzy subgroup of $\mathrm{G}$ then $(\operatorname{supp} \lambda, \mathrm{X}, \pi / \operatorname{supp} \lambda \times X$ is fuzzy topological transformation group.

Result 5.4 Consider the map $f: X \rightarrow X / \lambda$ given by $f(x)=\left[\lambda_{x}\right]$. Define a map $\phi: G \times X \rightarrow X / \lambda$ as $\phi\left(t,\left[\lambda_{x}\right]\right)=f(\pi(t, x))$. Then $(\phi, G, X / \lambda)$ is a fuzzy topological transformation group.

Proof. $\mathrm{f}$ and $\pi$ being continuous, we have $\phi$ is continuous. Now $\phi\left(0,\left[\lambda_{x}\right]\right)=f(\pi(0, x))=f(x)=\left[\lambda_{x}\right]$. Also $\phi\left(s, \phi\left(t,\left[\lambda_{x}\right]\right)\right)=\phi\{s, f(\pi(t, x))\}=\phi\left\{s,\left[\lambda_{\pi(t, x)}\right]\right\}=f\left\{\pi(s, \pi(t, x)\}=f\{\pi(s+t, x)\}=\phi\left(s+t,\left[\lambda_{x}\right]\right)\right.$. Thus (FGT1), (FGT2) and FGT3) are satisfied and hence $(\phi, G, X / \lambda)$ is a fuzzy topological transformation group.

In $\mathrm{X}$ define a relation $x \sim y$ if $\left[\lambda_{x}\right]=\left[\lambda_{y}\right]$. Then this relation is an equivalence. Denote the equivalence class of $\mathrm{x}$ by $[x]$. Let $\mathrm{g}$ be the canonical mapping from $\mathrm{X}$ to $\{[x]: x \in X\}$, i.e, $g(x)=[x]$. Equip $\{[x]: x \in X\}$ with the corresponding quotient topology. Then clearly this space is fuzzy homemorphic with the already introduced space $X / \lambda$, where the corresponding homeomorphism is $x \rightarrow\left[\lambda_{x}\right]$. We can denote both the spaces by the same notation $X / \lambda$. Consequently $(\phi, \mathrm{G}, X / \lambda)$, where $\phi: G \times X \rightarrow X / \lambda$ defined as $\phi(x)=g(\pi(t, x))$ is a fuzzy topological transformation group. Here $X / \lambda$ stands for the quotient space $\{[x]: x \in X\}$.

Conclusion : In this paper we have developed the notion of topological transformation group in fuzzy setting. We have attempted to extend most of the results of classical topological transformation group to this fuzzy setting. A topological transformation group is the basic structure in the study of topological dynamics. As the concept of orbit and orbit closure are fuzzified, it is expected that the other concepts of topological dynamics can be worked on in this setting.

Acknowledgement : The authors are thankful to the referees for their comments on the paper. The first author is grateful to the University Grants Commission, New Delhi, for the financial support provided for the work under a minor research project.

\section{References}

A. K. Katsaras, (1981), Fuzzy topological vector spaces 1, Fuzzy sets and systems, 685 -95.

Chu-hai Yu and Ji-liang MA, (1987), On fuzzy topological groups, Fuzzy sets and systems 23, 281-287.

Liu Ying-Ming and Luo Mao-Kang, (1997), Fuzzy topology, World Scientific.

M.H. Anvari, (2007), Transitivity and topological entropy on fuzzy dynamical systems through fuzzy observations, Proceedings of the 8th conference of 8th WSEAS international conference on fuzzy systems, Vol. 8 , 170-174.

M. Nadjafikhah and R.B. Chamazkoti, (2008), Fuzzy Lie Groups, Mathematical Sciences, Vol.2, No.2, 193-206.

N. Palaniappan, (2005), Fuzzy topology, 2nd ed., Norasa Publishing House.

Robert Ellis, (1969), Lectures on topological dynamics, W.A.Benjamin, INC.

Rajesh Kumar, (1993), Fuzzy Algebra, Vol. 1, University of Delhi Publication.

R. Lowen, (1976), Fuzzy topological spaces and fuzzy compactness, J. Math. Anal. Appl. 56, 621-633.

R. Lowen, (1977), Initial and Final topologies and fuzzy Tychonoff theorem, J. Math. Anal. Appl. 54, pp.11-21.

W.H. Gottschalk and G.A. Hedlund, (1955), Topological Dynamics, AMS. 